\title{
Electrochemical characterization of ZrTi alloys for biomedical applications
}

\author{
Georgiana Bolat $^{1,2}$, Javier Izquierdo ${ }^{2}$, Juan J. Santana ${ }^{3}$, Daniel Mareci ${ }^{1}$, Ricardo M. Souto ${ }^{2}$ \\ ${ }^{1}$ Faculty of Chemical Engineering and Environmental Protection, 'Gh.Asachi', Technical \\ University of Iasi, B-dul D. Mangeron, nr. 59, 700050 Iasi (Romania) \\ ${ }^{2}$ Department of Physical Chemistry, University of La Laguna, E-38205 La Laguna (Tenerife, \\ Canary Islands), Spain \\ ${ }^{3}$ Department of Process Engineering, University of Las Palmas de Gran Canaria, Campus \\ Universitario de Tafira, E-35017 Las Palmas de Gran Canaria (Gran Canaria, Canary Islands), \\ Spain.
}

\begin{abstract}
:
The electrochemical behaviour of three ZrTi alloys (Zr5Ti, Zr25Ti and Zr45Ti) in Ringer’s solution has been investigated. Their resistance against localized corrosion has been determined from cyclic potentiodynamic polarization (CCP) and electrochemical impedance spectroscopy (EIS) measurements, whereas scanning electrochemical microscopy (SECM) was applied to investigate the local reactivity of the passive films developed on the materials, and scanning electron microscopy (SEM) was employed to characterize the surface morphology of the alloys subjected to anodic polarization. An increased reactivity could be detected with SECM when the metal samples were polarized at $+0.50 \mathrm{VSHE}$, though the extent of this feature greatly depended on the nature of the metallic material. At $37{ }^{0} \mathrm{C}$, the Zr5Ti alloy was susceptible to localized corrosion. Though Zr25Ti alloy presented rather low pitting potential, the spontaneous corrosion potential of the material was sufficiently negative to require overpotentials around $600 \mathrm{mV}$ for breakdown to occur. Finally, the Zr45Ti alloy exhibited a larger passive range in the polarization curve, and it was resistant to localized corrosion.
\end{abstract}

Keywords: ZrTi alloys, corrosion resistance, cyclic potentiodynamic polarization curves, EIS, scanning electrochemical microscopy, SEM.

\section{Introduction}

Titanium and its alloys (especially Ti6Al4V) are widely used in restorative surgery due to their good biocompatibility and excellent corrosion resistance in physiological media [1-3]. Yet corrosion remains a concern because the protective passivating oxide layers that separate the metal from the environment are not perfect insulating barriers. Transient events related to localized depassivation have been observed for these materials in vitro [4-6], and they may release metal ions 
in the surroundings. There are reports in the literature describing several cases of extensive metallosis and necrosis in periprosthetic tissues of failed cemented Ti6Al4V prostheses [7,8], as well as local and systemic effects of corrosion products [9-14]. Thus, there is increasing awareness on the presence of vanadium in long term implants because this element is toxic both in the elemental state and in its oxides $[15,16]$. Additionally, the association between $\mathrm{Al}$ and Alzheimer disease has also been suggested $[17,18]$.

The toxicity of vanadium pushes forward the development of new Ti-based alloys to replace Ti6Al4V. In particular, new $\beta$-type titanium alloys with biomechanical compatibility, low modulus and biochemical compatibility are sought $[19,20]$. $\beta$-stabilizing elements, such as Mo, Ta, Zr and Sn, are judged to be non-toxic and non-allergenic [21], and are thus selected as safe alloying elements to titanium. Zirconium and titanium are in the same group in the periodic table of elements, and are known to have similar chemical properties [22]. Both metals usually have their surfaces covered by passivating thin oxide films that are spontaneously formed in air or in electrolytes at open circuit $[23,24]$. Though $\mathrm{Zr}$ offers superior corrosion resistance over most other metals in different electrolytes [25], this metal is susceptible to localized corrosion induced by $\mathrm{Cl}^{-}$ions [26,27]. In terms of biomaterial applications, the most inconvenient characteristics are related to degradation, which occurs due interaction of the material with body or physiological fluids that typically present concentrations of ca. 1 wt.\% $\mathrm{NaCl}$ [28]. In this context, $\mathrm{Yu}$ and Scully have reported that Ti13Nb13Zr alloy has an extremely high resistance to active and passive dissolution in oxidizing acids [29]. The binary ZrTi system exhibits complete solid solution, thus efforts can be directed to finding an adequate ratio for the alloying of $\mathrm{Zr}$ with $\mathrm{Ti}$ that would produce a material with decreased or even hindered susceptibility to localized corrosion compared to pure zirconium. Electrochemical characterization of the new materials in simulated physiological environments is an important tool for this material selection process.

The current paper reports on the susceptibility to localized corrosion exhibited by various ZrTi alloys with different compositions during exposure to Ringer's solution. For the sake of comparison, titanium grade 1 was also investigated in the same experimental conditions. Electrochemical characterization was performed using cyclic potentiodynamic polarization (CPP) and electrochemical impedance spectroscopy (EIS) techniques with the objective to determine the potential ranges for the stability of the materials, and to quantify the resistance of the passive films developed on them. Additionally, scanning electrochemical microscopy (SECM) was applied to monitor the local reactivity of the passive films exposed to the test environment. Probe approach curves and scan arrays were recorded at selected areas for this investigation. 


\section{Materials and methods}

\subsection{Materials and sample preparation}

Three ZrTi experimental alloys were investigated. The code names and chemical compositions of the alloys are shown in Table 1. For comparison, titanium grade 1 was also considered. The non alloyed Ti samples were obtained from a bar stock in annealed state. The structural characterization of this metal was detailed elsewhere [30].

The samples were ground with a sequence of 400 to 4000 grit emery paper and subsequently polished with alumina suspension to attain mirror-appearance, washed with Milli-Q deionised water (18.2 M $\Omega$ ), ultrasonically cleaned and degreased in ethanol, and dried in air. Tests specimens were embedded in a polytetrafluoroethylene (PTFE) holder specifically designed to facilitate connection to a rotating disc electrode holder (model EDI 101T, Radiometer Analytical). A polymeric resin was used to ensure a tight seal between the specimen and the PTFE holder, in order to avoid the occurrence of crevice corrosion [31]. The exposed surface of the materials to the test environments were discs of $0.196 \mathrm{~cm}^{2}$ area. The same arrangement was employed for the SECM experiments, though in this case the mount containing the metals was placed at the bottom of the small electrochemical cell with the metal facing upwards to the electrolyte. In this way, scans could be performed both vertically (approach curves) and parallel (array scans) to the sample surface.

\subsection{Test corrosion medium}

Electrochemical tests were conducted in aerated physiological Ringer's solution whose composition was: $\mathrm{NaCl}: 8.6 \mathrm{~g} / \mathrm{L}, \mathrm{KCl}: 0.3 \mathrm{~g} / \mathrm{L}, \mathrm{CaCl}_{2}: 0.48 \mathrm{~g} / \mathrm{L}$. The $\mathrm{pH}$ of this solution was 6.8. SECM experiments were performed in Ringer's solution containing $0.5 \mathrm{mM}$ ferrocene-methanol as the redox mediator. Electrolyte solutions were prepared using analytical grade chemicals and Milli-Q deionised water.

\subsection{Electrochemical measurements}

The test specimens were placed in a glass corrosion cell, which was filled with freshlyprepared electrolyte. A saturated calomel electrode was used as the reference electrode, and a platinum coil as the counter electrode. The potentials in this paper are reported versus the standard hydrogen electrode (SHE). The temperature of the electrochemical cell was maintained at $37{ }^{0} \mathrm{C}$.

Electrochemical measurements were performed using a potentiostat manufactured by PAR (Model 263A, Princeton Applied Research, Princeton, NJ, USA). The instrument was controlled by a 
personal computer and specific software (Electrochemistry Power Suite, PAR, USA). The open circuit potentials of the materials attained stable values after 1 hour immersion in Ringer's solution $\left(E_{c o r r}\right)$. Therefore, measurement of cyclic potentiodynamic polarization curves (CPC) was initiated after 1 hour exposure to the test environment. The tests were conducted by scanning the potential at $0.5 \mathrm{mV} \mathrm{s}^{-1}$ from $-0.76 \mathrm{~V}_{\text {SHE }}$ to $+1.24 \mathrm{~V}_{\text {SHE }}$, followed by scanning the potential in the negative direction down to -0.26 V SHE. Using PowerCorr software (PAR, USA), the cyclic potentiodynamic polarization curves were plotted, and the corresponding values for the open circuit potential ( $\left.E_{c o r r}\right)$ and the corrosion current density $\left(j_{c o r r}\right)$ were determined. From the cyclic potentiodynamic polarization curves, the passivation current density $\left(j_{p a s s}\right)$, breakdown potential ( $\left.E_{b p}\right)$, and repassivation potential $\left(E_{r p}\right)$ values were also obtained, which are indicators of the stability of the passive regime of the materials.

Electrochemical impedance spectroscopy (EIS) measurements were performed on the samples subjected to various potential conditions while immersed in Ringer's solution. A lock-in amplifier (Model 5210, Princeton Applied Research, Princeton, NJ, USA) was employed for EIS measurements using $10 \mathrm{mV}$ amplitude of sinusoidal voltage over a frequency range extending from $100 \mathrm{kHz}$ to $10 \mathrm{mHz}$. First, EIS spectra were acquired at the open circuit potential attained by the samples after 1 hour immersion in Ringer's solution. Next, the potential of the sample was set at $+0.54 \mathrm{VSHE}$ for $30 \mathrm{~min}$ before recording the EIS data of the polarized sample. The EIS experimental data were analyzed in terms of equivalent circuits (EC) using ZsimpWin 2.00 software [32].

All electrochemical tests were repeated three times to ensure reproducibility of the measurements.

\subsection{Scanning electrochemical microscopy (SECM)}

The SECM experiments were performed using a Model 900 Scanning Electrochemical Microscope from CH Instruments (Austin, TX, USA), controlled with a personal computer. The specimens were mounted horizontally facing upwards at the bottom of a cell made of polytetrafluoroethene, which was equipped with an $\mathrm{Ag} / \mathrm{AgCl} / \mathrm{KCl}(3 \mathrm{M})$ reference electrode and a platinum counter electrode, all set up in. For the sake of comparison, potential values were referred to the standard hydrogen electrode (SHE). The electrochemical cell was located inside a Faraday cage. Tip microelectrodes were made from $10 \mu \mathrm{m}$ platinum wires sealed in glass. $0.5 \mathrm{mM}$ ferrocenemethanol was added to the Ringer's solution to act as electrochemical mediator at the tip. To enable the oxidation of the ferrocene-methanol the tip was kept at a constant potential of $+0.70 \mathrm{VSHE}$. In some experiments the metal specimens were polarized using the bipotentiostat associated with the SECM. 
The micromanipulator stand of the SECM instrument was used to hold the microelectrode in place. Measurements were performed both vertically (approach and retreat curves), and parallel (array scans) to the sample surface at a height of $10 \mu \mathrm{m}$. Temperature control was not performed in the SECM, and data were recorded at ambient temperature (around $20{ }^{\circ} \mathrm{C}$ ).

\subsection{SEM}

The surface morphology of the specimens after electrochemical testing was observed by scanning electron microscopy. SEM observations were made using a Quanta 200 (FEI, Hillsboro, OR, USA) operated at an accelerating voltage of $30 \mathrm{kV}$.

\section{Results and discussion}

Figure 1 shows typical cyclic potentiodynamic polarization curves for pure Ti and the ZrTi alloys plotted in a semi-logarithmic presentation. They were recorded after 1 hour immersion in Ringer's solution at $37^{\circ} \mathrm{C}$. Average open circuit potential $\left(E_{c o r r}\right)$ and corrosion current density $\left(j_{\text {corr }}\right)$ values determined from the polarization curves are listed in Table 2 for the various materials tested.

None of the materials exhibited a distinctive active-passive transition in the polarization curves following the Tafel region, but they entered directly into a stable passive regime. Next, the polarization curves measured for the three ZrTi alloys (namely Zr45Ti, Zr25Ti and Zr5Ti) exhibited positive hysteresis loops in the cyclic polarization curves, indicating that these materials are susceptible to localized corrosion (cf. Figure 1). In contrast, the pure titanium specimen showed no positive hysteresis loop in the cyclic polarization curve.

Though the ZrTi alloys exhibited common features in their CPC, it must be noticed that their corrosion resistance greatly varied between them as result of the different content of titanium in the materials. The susceptibility of an alloy to localized corrosion in a given environment can be characterized in terms of the breakdown potential $\left(E_{b p}\right)$ and repassivation potential $\left(E_{r p}\right)$, relative to the corresponding open circuit potential value $\left(E_{c o r r}\right)$. The potential range comprised between the open circuit potential and $E_{b p}$ represents the passivity zone, where corrosion is small or even insignificant. Thus, the difference $E_{b p}-E_{c o r r}$ can be adopted as a measure of the passive range of the material [33-35]. Additionally, the extent of the area covered by the hysteresis loop (i.e., the difference between $E_{r p}$ and $E_{c o r r}$ values) is a direct measure of the susceptibility of the material to localized corrosion [34].

Table 2 lists the values of the breakdown $\left(E_{b p}\right)$, and repassivation $\left(E_{r p}\right)$ potentials found for the materials under investigation, together with the values calculated for the differences $E_{b p}-E_{c o r r}$ and $E_{r p}-E_{c o r r}$. Based on these data, the corrosion resistance of the various ZrTi alloys immersed in 
Ringer's solution can be characterized. The CPC for the alloy Zr5Ti exhibits both the more negative breakdown potential value and the narrowest potential range for passivity. These facts indicate that this alloy exhibits the greater susceptibility to localized corrosion. The electrochemical behaviour of Zr5Ti resembles that of pure zirconium [36], and the titanium content in the alloy is not enough to produce a significant improvement in the resistance of the material against localized corrosion in Ringer's solution at $37^{\circ} \mathrm{C}$.

Alloys containing higher amounts of titanium exhibit longer potential ranges for the passive regime, due to the shift of their breakdown potentials towards more positive values. This feature is readily observable for the Zr25Ti alloy as compared to Zr5Ti. Yet the more positive potential for breakdown of the passive film attained by Zr25Ti in Ringer's solution is not be regarded safe enough for this material to be proposed for biomedical application. The reason for this consideration arises from reports describing the exposure of Ti to positive potentials up to $+0.64 \mathrm{~V}_{\text {SHE }}$ in the human body [37]. Though similar information regarding the ZrTi alloys is not available in the scientific literature yet, it can be estimated that similar potential values may occur as well. Thus, further addition of titanium to zirconium is still necessary to obtain a zirconium-based material that may be considered for biomedical application. The electrochemical behaviour of Zr45Ti is more promising. The passive zone now extends for almost $1.0 \mathrm{~V}$, and a higher positive breakdown potential (ca. $+0.94 \mathrm{VSHE}$ ) is exhibited by this alloy, that is, $0.30 \mathrm{~V}$ more positive than the highest value recorded in the human body until now [37].

Though the trend exhibited by the pitting potential values can be related to the susceptibility of the material to localized corrosion, the possible occurrence of breakdown also depends on the surface condition of the metal in the environment, which results in variations in the value of the open circuit potential. In this way, the values of $E_{b p}-E_{c o r r}$ provide a more reliable measure of the breakdown resistance [34]. Indeed, an alloy with a particular surface condition may exhibit a small $E_{b p}$ and yet have a sufficiently negative $E_{c o r r}$, that the difference between them is big. This was the case for Zr25Ti alloy exposed to Ringer's solution, namely it exhibited a small Ebp value (around $+0.53 \mathrm{VSHE}$ ), but the difference $E_{b p}-E_{\text {corr }}$ exceeded $0.60 \mathrm{~V}$. In this study, larger passive ranges and more positive corrosion potentials were found for the alloys with a higher titanium contents. In fact, pure titanium exhibited the more positive value for $E_{c o r r}$, and no breakdown potential of the passive film was found for potential excursions up to $+1.24 \mathrm{~V}$ SHE.

The values of the passive current density ( $j_{\text {pass}}$ ) were also determined from the potentiodynamic polarization curves. They were taken from the anodic branch of the potentialcurrent density plots at around the middle of the corresponding passive regions for each material, and they are listed in Table 2. They were small for the Zr5Ti and Zr25Ti alloys, namely in the range of 
$0.5-1 \mu \mathrm{A} \mathrm{cm}{ }^{-2}$, whereas for Zr45Ti greater passivity currents were found, possibly related to the development of a thicker oxide film. Estimates of the thickness of the passive films have been derived from electrochemical impedance data.

Electrochemical impedance spectroscopy (EIS) measurements were performed both under open circuit potential conditions and under polarization at $+0.54 \mathrm{~V}$ sHE. This polarization value was chosen to account for the eventual development of positive potentials in the human body as described before. The EIS spectra of the non-polarized samples was measured after they attained a stable open circuit potential in Ringer's solution, whereas the measurements for the polarized samples were initiated after application of the potential value $E=+0.54 \mathrm{~V}_{\text {SHE }}$ for $30 \mathrm{~min}$.

Figure 2 depicts the Bode impedance plots for titanium and the ZrTi alloys under open circuit potential conditions in aerated Ringer's solution at $37{ }^{\circ} \mathrm{C}$. From a cursory observation of the spectra it can be deduced that they exhibit behaviours consistent with the formation of a thin passive oxide film on the surface of the materials, i.e., a near capacitive response illustrated by a phase angle greater than $-45^{0}$ over a wide frequency range. The passive behaviour of pure titanium in Ringer's solution can be described by one time constant. The shape and the values obtained are very similar to those values previously reported for titanium grade 1 immersed in phosphate buffer solution by Pan et al. [38]. In their work they extended the low frequency range of the measurements down to $5 \mathrm{mHz}$, and though they fitted their spectra considering two time constants, the resistance of the outer layer was very small for its impact to be noticed on the shape of the Bode plots. The same system was later investigated by Aziz-Kerrzo et al. [39], though using high purity titanium (99.999\%). In this case, two time constants were observed for frequencies below $1 \mathrm{~Hz}$, and higher resistance values were estimated for the outer layer of the passive film. The variation could be assigned to the development of a more porous outer layer due to impurities in the material in our case.

ZrTi alloys observed by EIS at their open circuit potential values can also be described by one time constant. Since the low frequency range limit of our measurements was set at $10 \mathrm{mHz}$, the occurrence of a second time constant below that frequency can not be discarded. Yet, it should be noticed that most Ti-based alloys exhibiting two time constants have the transition between them in the 0.1-1.0 Hz range [39]. Therefore, the spectra were analyzed in terms of the equivalent circuit proposed for a sealed passive layer [38] that is depicted in Figure 3A. It assumes that the corrosion of the passive metal is hindered by an oxide film that acts as a barrier-type compact layer. The equivalent circuit consists of the parallel combination terms $\left(R_{p L} Q_{p L}\right)$ in series with the resistance of the solution ( $R_{s o l}$ ) occurring between the sample and the reference electrode. The parameters $R_{p L}$ and $Q_{p L}$ describe the properties of the passive films formed on these metallic materials, respectively the resistance and capacitance of the compact oxide layers. 
For fitting the spectra, a constant phase element (CPE) was used instead of a pure capacitance because of the non-ideal capacitive response due to the distributed relaxation feature of the passive oxide films, which is observed as a depressed semicircle when the spectra are plotted in the corresponding Nyquist diagrams (not shown here). The impedance representation of CPE is given as:

$$
Z_{(C P E)}=\frac{1}{Y_{0}(j \omega)^{n}}
$$

where $\omega$ is the angular frequency and $Y_{0}$ is a constant, and the value of the exponent $n$ indicates the deviation from ideal capacitive behaviour (e.g., when $n=1$ ). In general, the CPE is given as both capacitance $C$, given in units $s^{n} \mathrm{~S}$, and factor $n$. Exponent values smaller than 1 arise from surface roughness of the oxide films. Yet the values were always greater than 0.8 , which may indicate a rather smooth surface of the passive layers. Analysis of the impedance spectra in terms of this equivalent circuit allowed for the parameters $R_{p L}$ and $Q_{p L}$ to be determined, and they are presented in Table 3. The fits provided similar values for $R_{\text {sol }}$ in all cases, namely $70 \pm 5 \Omega \mathrm{cm}^{2}$. The high quality of the fitting is readily seen from the good match between the measured and the fitted spectra, which are presented as solid lines together with experimental data on the Bode plots in Figure 2.

From the capacitance value, the oxide film thickness can be known using the parallel-plate capacitor equation:

$$
C=\frac{\varepsilon \varepsilon_{0} A}{d}
$$

where $\varepsilon$ is the dielectric constant of the oxide and $\varepsilon_{0}$ the dielectric permittivity of vacuum, $A$ is the effective area, and $d$ the thickness of the oxide layer [40]. Since the values of the capacity of the passive oxide layer can be derived from CPE parameters using the following equation [11]:

$$
C=\left(R^{1-n} Q\right)^{1 / n}
$$

the thickness of the oxide films formed on the titanium alloys could be estimated. To that end, $\varepsilon=65$ was assumed, which is the value for $\mathrm{TiO}_{2}$ [41], and the surface roughness factor as unity. The thicknesses of the oxide films formed on the different materials are given in Table 4. The value obtained for pure titanium (namely $4.5 \mathrm{~nm}$ ) agrees well with previous reports in the literature [41]. Thinner films are formed when less Ti is present in the alloy, and the thickness for Zr5Ti, $2.9 \mathrm{~nm}$, is close to that of pure zirconium spontaneously covered in air $(2.5 \mathrm{~nm})[42,43]$. Therefore, the values reported in Table 4 are regarded to be a good estimate of the effect of composition on the thickness of the oxide films, though they were based on the assumption of $\varepsilon=65$ to apply to the zirconium alloys, which may not be the case. 
High impedance values, in the order of $10^{6} \Omega \mathrm{cm}^{2}$, were obtained in the medium and low frequency ranges for ZrTi alloys, suggesting high corrosion resistance of the materials in the Ringer's solution. The presence of protective passive layer provided the high corrosion resistance of the materials in this aqueous environment. The contribution of charge transfer resistance on the impedance data is regarded negligible for all the alloys. Additionally, it can be observed in Table 3 that the value of the passive layer resistance obtained at open circuit potential, $R_{p L}$, increases as the contents of $\mathrm{Ti}$ in the alloys are made smaller (i.e., $500 \mathrm{k} \Omega \mathrm{cm}^{2}$ for pure Ti, and $2,100 \mathrm{k} \Omega \mathrm{cm}^{2}$ for Zr5Ti). This feature demonstrates that the spontaneously formed passive layer on ZrTi alloys becomes more resistant when the amount of titanium in the alloy decreases.

EIS measurements were also performed when the samples were polarized at $+0.54 \mathrm{VSHE}$, and the corresponding spectra are given in Figure 4. In this case, differences can be observed between the spectra which vary significantly among the tested materials. Two different situations can be distinguished by considering the resistance values attained in the low frequency range, namely: (1) pure Ti and the Zr45Ti alloy display higher resistances under anodic polarization than at the open circuit potential (respectively 2,900 and $500 \mathrm{k} \Omega \mathrm{cm}^{2}$ for Ti, and 1,400 and $800 \mathrm{k} \Omega \mathrm{cm}^{2}$ for Zr45Ti); and (2) Zr25Ti and Zr5Ti present the opposite trend (polarized Zr25Ti exhibits a value of $500 \mathrm{k} \Omega$ $\mathrm{cm}^{2}$ whereas it amounted $1,200 \mathrm{k} \Omega \mathrm{cm}^{2}$ at the OCP, and it could not be determined for the polarized Zr5Ti). Enhanced resistance is displayed by both Ti and the alloy with the highest titanium content, which is an indication of additional growth of the oxide films favored by anodic polarization. In this case, the Bode-phase plots show two relaxation constants, i.e. two peaks are observed in the Bodephase plots. They can be satisfactorily fitted with the equivalent circuit shown in Figure 3B, which can be regarded as an electrical representation of a two-layer model of the oxide film consisting of a barrier-type compact inner layer and a relatively porous outer layer [44]. The new components in the EC are the resistance and the capacitance associated with the outer layer.

Conversely, smaller resistances are exhibited by Zr5Ti and Zr25Ti when polarized at this anodic potential, indicating a thinning of the oxide film or even losses of the film continuity due to localized breakdown. Yet some differences can be observed between the two materials. The two time constants remain observable in the spectrum measured for polarized Zr25Ti, and the reported decrease in the resistance value determined in the low frequency limit is rather small. Conversely, Zr5Ti shows only one time constant and the resistance is decreased by almost 2 orders of magnitude. It is interesting to notice that the remarkable difference in shape between the spectra for this alloy and the other materials could be correlated with variations in the morphology of the samples retrieved after recording the EIS spectra. Figure 5 shows the corresponding SEM micrographs for the 
three ZrTi alloys and for pure titanium. The extent of the corrosion reaction on the polarized Zr5Ti sample spanning over the time required to record the EIS data becomes observable in Figure 5A. The surface roughening developed for this material was enough for the polishing lines not to be observed any longer. That is, the oxide film spontaneously formed over the surface of this alloy at the OCP during immersion in Ringer's solution, became unstable upon application of an anodic potential of $+0.54 \mathrm{~V}$ SHE. The underlying metal is then directly exposed to the electrolyte and corrodes in the aqueous environment.

The onset of corrosion during anodic polarization at $+0.54 \mathrm{~V}_{\mathrm{SHE}}$ in Ringer's solution also occurred in the case of Zr25Ti, though in this case the polishing lines can still be observed (see image in Figure 5B). The nucleation of corrosion pits of micrometric dimensions is observed, as well as some roughening of the surface, though certainly in a smaller scale than for Zr5Ti. Therefore, the protective oxide film is still present on the surface of the material, though localized breakdown leading to pitting corrosion occurs, which is a very dangerous type of degradation process because the corrosive attack effectively penetrates in the bulk of the material. Conversely, no attack was observed in the case of Zr45Ti and pure titanium (see micrographs C and D in Figure 5). The passive films formed on the surface of these two materials effectively protect the underlying metallic matrix even at the anodic polarization of $+0.54 \mathrm{~V}_{\mathrm{SHE}}$. In summary, addition of titanium to zirconium leads to the formation of oxide films on the surface of the metallic material that more efficiently resist the onset of localized forms of corrosion.

The EIS spectrum measured for Zr25Ti while polarized at $+0.54 \mathrm{~V}_{\text {SHE }}$ could still be satisfactorily fitted with the two-layer model presented in Figure 3B, though the less resistant system is evidenced by the smaller value determined for $R_{p L}$, the resistance of the inner passive layer. Finally, a different electrical model was required to fit the spectrum obtained for the polarized Zr5Ti alloy. In this case, the equivalent circuit is presented in Figure 3C, which is typical for a corroding metal. Thus, under such condition, the inner compact passive layer was no longer present to protect the metal from the environment. Extensive degradation of the passive layer occurred for Zr5Ti alloy polarized at +0.54 VsHe in Ringer's solution.

In general, a very good agreement between the fitted and the experimental data was also achieved for the polarized samples. Table 3 shows the results of the fits. The parameters $R_{c t}$ and $Q_{d L}$ account for the properties of the reactions at the passive layer/solution interfaces and determine the impedance behaviour in the high frequency range of the spectrum. The capacitance values associated with $Q_{d L}$ for all the samples are typical of those related to the double layer capacitance of passive oxide layers [45-48]. Therefore $R_{p L}$ and $Q_{p L}$ parameters describe the properties for passive layer. The 
resistance of passive layer of ZrTi alloys polarized at +0.54 VsHE in Ringer's solution gradually decreases with the Zr content, implying that the corrosion resistance of ZrTi alloy decreases for greater Zr contents.

Scanning microelectrochemical measurements were also performed to spatially resolve different electrochemical properties of the oxide films originating from their heterogeneous composition. Scanning electrochemical microscopy (SECM) in the feedback mode, using ferrocenemethanol as redox mediator, was chosen for this purpose. In this operation mode, the current arising from the oxidation of the ferrocene-methanol to the ferrocinium ion at the SECM tip was monitored. To secure that the tip would always operate under diffusion-controlled regime, the tip potential was set at +0.70 VSHE for the SECM measurements.

The tip-substrate distance was established by recording Z-approach curves towards the surface of the metallic materials, while they were effectively maintained at their $E_{\text {corr }}$ value in the test electrolyte. Once located the surface, the tip was retracted $10 \mu \mathrm{m}$ from the surface, and scan arrays covering an area of $250 \mathrm{x}$ $250 \mu \mathrm{m}^{2}$ were registered by moving the microelectrode in an $X Y$ plane parallel to the sample. The microelectrode was subsequently moved back to the bulk solution while still measuring the current response related to ferrocene oxidation, and the retracting curve was obtained. This procedure was then repeated while the metallic substrate was polarized at +0.50 VSHE. Figure 6 displays the Z-approach and Zretreat plots measured for Ti and the ZrTi alloys. Dimensionless tip currents are represented in the graphs as a function of the tip-substrate distance. $i$ / $i_{\text {lim }}$ values were obtained by dividing the currents at each location by the diffusion-limited current measured in the bulk electrolyte (i.e. sufficiently far from the surface for geometrical effects to be experienced).

Changes in the faradaic current measured at the tip are observed when it is located in close proximity to a surface under investigation. If the substrate is a conductive surface that can exchange electrons with the chemical species formed at the tip, the redox mediator species can be regenerated. Regeneration of the redox mediator would result in a fast increase of the currents measured at the tip when it approaches the surface of the substrate. Thus, this positive feedback effect would produce $i$ / $\dot{i}_{\text {lim }}$ values greater than one. Conversely, a negative feedback effect is observed when the tip approaches an insulating substrate due to geometric hindrance for the diffusion of the reacting mediator. The tip current steadily diminishes when the tip approaches the substrate, and $i / i_{l i m}$ values smaller than one occur in this case.

There was a continuous decrease of the current measured at the tip as the tip moved towards the substrates for all the materials when they were polarized at +0.50 VsHE. This negative feedback behaviour indicates that the oxide layers formed on the surfaces act as a barrier towards electron transfer reactions. The same dependence between the tip current and the tip-substrate distance was observed when the tip was 
removed from the surface for all of the materials except Zr45Ti. For this alloy, activation of the surface was observed, and this effect will be discussed later.

Though a negative feedback regime also describes all the vertical lines measured at $E_{\text {corr }}$, some differences with the behaviour described for the polarized samples should be noticed. Firstly, the faradaic current measured at the tip initially increased with respect to the limiting diffusion value $i_{\text {lim }}$ when the tip experienced the influence of the substrate. Such increase occurred rather slowly with variations in the tipsubstrate distance. In this way, dimensionless current values bigger than one were observed over a rather extended interval of normalized distances. Secondly, a fast decrease of the faradaic current occurs when the tip was located in a close proximity to the substrate, namely for $d / a \leq 3$. Therefore, at the smallest tipsubstrate distances, hindered diffusion of ferrocene-methanol towards the tip became the main contribution to the overall current. But at intermediate distances, some regeneration of the redox mediator occurred on the thinner oxide films formed at the more negative potentials corresponding to the $E_{c o r r}$ for each material. This behaviour has also been found for other titanium alloys containing either molybdenum or tantalum [49]. Regeneration of the redox mediator is more efficient on the oxide film formed on pure titanium, and the hindrance of diffusion occurring at the smallest tip-substrate distance is effectively overcome by the enhanced rate of ferrocinium ion reduction at the oxide covered metal. For this metal, $i / i_{\text {lim }}>1$ at all tipsubstrate distances. In summary, from the point of view of the shape of the Z-approach and Z-retreat curves, it can be deduced that the conductivity and the electrochemical reactivity of the passive films formed on these materials is influenced by their elemental composition because they affect the composition of the oxide film. Additionally, the behaviour of the alloys under spontaneously-developed electrical conditions can be significantly different from those generated during conventional electrochemical experiments, which are based on the application of a potential perturbation to the system under study, and this is applied to the overall material surface exposed to the test solution.

SECM images obtained for the samples either maintained at their corresponding $E_{c o r r}$, or under polarization at $+0.50 \mathrm{~V}_{\mathrm{SHE}}$, are given in Figures 7 and 8, respectively. Titanium showed some heterogeneity even at the $E_{\text {corr }}$ (see Figure 7A). That is, tip currents greater than ilim were observed at certain locations. This feature is due to facilitated regeneration of ferrocene-methanol at those areas, whereas hindered electron transfer occurs in the remaining surface. When the substrate was polarized at $+0.50 \mathrm{~V}$ SHE (cf. Figure 8A), no regions with normalized tip currents greater than 1 could be found. In this case, the SECM image exclusively reveals the topography of the sample. This result is not surprising, for the anodic potential applied to the substrate is already too positive for the electroreduction of ferrocinium ions. Then, the changes in the current values from top to bottom exclusively reflect some unavoidable tilt of the sample as observed in Figure 7A, though the main features in the image correspond to variations in the electrochemical reactivity of the investigated surface. 
Featureless SECM images were obtained above Zr25Ti and Zr5Ti for both the unbiased and the polarized substrates (see Figures 7C, 7D, 8C, and 8D), thus they exclusively provide topographic information. The passive films formed on these alloys at the $E_{c o r r}$ are insulating enough for electron transfer not to occur at them. Yet, variations in the thickness of the passive films formed at the different potentials account for the variations in the average tip currents measured in each case. That is, higher faradaic currents for ferrocene-methanol oxidation are measured at the $E_{\text {corr }}$ of each metal, which corresponds to the less insulating surfaces resulting from thinner oxide films.

A special case is provided by the SECM maps measured for Zr45Ti alloy, which are displayed in Figures 7B and 8B. Negative feedback behaviour is clearly observed in the image recorded at the $E_{c o r r}$ for all the scanned area, though some locations are slightly more active (i.e. leading to higher tip currents). However, when the sample was polarized at $+0.50 \mathrm{VsHE}$, a sudden increase of the tip current values was observed, indicating that the metal surface became electrochemically activated. This new electrochemical behaviour was also observed in the Z-retreat curve subsequently registered, and remained during the measurement of a new Z-approach curve, (the two curves are shown in Figure 9). Even more striking is the observation that the apparent electrochemical activation of the surface affected the tip currents for tipsubstrate distances as large as $200 \mu \mathrm{m}$. Because the potential applied to the substrate is not negative enough to reduce the ferrocinium ions, this phenomenon cannot be attributed to a positive feedback behaviour. To our knowledge, such a phenomenon has not been reported in the scientific literature yet, and its origin cannot be explained at this stage. Further investigations using other redox mediators are currently undertaken at our laboratory to investigate this effect.

\section{Conclusions}

The electrochemical behaviour of three ZrTi alloys has been characterized in Ringer's solution $(\mathrm{pH}=6.8)$ in order to investigate their potential use as biomaterials to replace Ti-based alloys containing $\mathrm{Al}$ and $\mathrm{V}$. The development of passivity for all the materials could be deduced from the potentiodynamic polarization plots, and the corrosion resistance of ZrTi alloys in Ringer's solution results from the formation of a passive film on the surface of these materials. The current densities at the OCP, which are directly proportional to the corrosion rates of the materials, are observed to increase with greater titanium contents in the ZrTi alloys.

The addition of titanium to zirconium reduces the susceptibility of the later towards localized corrosion, as indicated by the shift of the breakdown potentials to more positive values. Though a sufficiently big overpotential (ca. $+0.60 \mathrm{~V}$ ) for film breakdown is already attained by $\mathrm{Zr} 25 \mathrm{Ti}$, the 
pitting potential of this alloy is more negative than the potential values that may occur in the human body [37]. Higher titanium contents in the alloys are required to ensure sufficient stability of the passive films developed on the ZrTi alloys for biomedical application.

EIS data show that the passive films behave as an insulating barrier towards electron transfer at the surface of the alloys, and this effect is more effectively provided by the ZrTi alloys compared to pure $\mathrm{Ti}$ when the oxide layers were spontaneously formed at their corresponding $E_{\text {corr }}$ values. But this effect could not be effectively maintained for alloys with titanium contents smaller than 45 wt.\% due to thickening and eventual breakdown of the passive oxide layers due to hazardous localized corrosion phenomena.

Localized differences in electrochemical reactivity of the materials could be established from SECM measurements. Though the passive films behave as an insulating barrier towards electron transfer at the surface of the alloys, this effect is more effectively provided by the ZrTi alloys compared to $\mathrm{Ti}$ when the oxide layers were spontaneously formed at their corresponding OCP values. Anodic polarizations at potentials reported to be achieved in the human body for Ti implants only led to thickening of the passive oxide layers without electrochemical activation due to hazardous localized corrosion reactions.

\section{Acknowledgements:}

This work was supported by the Romanian CNCS Program, under project PN-II-ID-PCE-2011-30218, No. 266, and the Ministerio de Ciencia e Innovación (MICINN, Madrid, Spain) and the European Regional Development Fund (Brussels, Belgium) under Grant Number CTQ200912459/PPQ. A Research Training Grant awarded to J.I. by the MICINN (Programa de Formación de Personal Investigador) is gratefully acknowledged. Thanks are due to Zirom S.A. (Giurgiu, Romania) for kindly providing the ZrTi alloys used in this work.

\section{References}

[1] D.F. Williams, Biocompatibility of clinical implant materials, in: Biocompatibility of clinical impact materials, vol. 1, D.F. Williams (Ed.); CRC Press, Boca Raton, FL, 1981, p. 9.

[2] P. Kovacs, J.A. Davidson, Chemical and electrochemical aspects of the biocompatibility of titanium and its alloys, in: Medical applications of titanium and its alloys: the materials and biological issues, S.A. Brown, J.E. Lemons (Eds.); ASTM, West Chonshohocken, 1996, p. 163.

[3] M. Geetha, A.K. Singh, R. Asokamani, A.K. Gogia, Ti based biomaterials, the ultimate choice for orthopaedic implants - A review, Progress in Materials Science 54 (2009) 397-425. 
[4] G.T. Burstein, R.M. Souto, Observations of localized instability of passive titanium in chloride solution, Electrochimica Acta 40 (1995) 1881-1888.

[5] R.M. Souto, G.T. Burstein, A preliminary investigation into the microscopic depassivation of passive titanium implant materials in vitro, Journal of Materials Science: Materials in Medicine 7 (1996) 337343.

[6] G.T. Burstein, C. Liu, R.M. Souto, The effect of temperature on the nucleation of corrosion pits on titanium in Ringer's physiological solution, Biomaterials 26 (2005) 245-256.

[7] C.P. Case, V.G. Langkamer, C. Jamec, M.R. Palmer, A.J. Kemp, P.F. Heap, L. Solomon, Widespread dissemination of metal debris from implants, Journal of Bone \& Joint Surgery 76B (1994) 701-712.

[8] I. Milošev, V. Antolič, A. Minovič, A. Cör, S. Herman, V. Pavlovčič, P. Campbell, Extensive metallosis and necrosis in failed prostheses with cemented titanium-alloy stems and ceramic heads, Journal of Bone \& Joint Surgery 82B (2000) 352-357.

[9] A. Balamurugan, S. Rajeswari, G. Balossier, A.H.S. Rebelo, J.M.F. Ferreira, Corrosion aspects of metallic implants - An overview, Materials and Corrosion 59 (2008) 855-869.

[10] M.V. Popa, E. Vasilescu, P. Drob, D. Mareci, J.M. Calderon Moreno, S. Ivanescu, C. Vasilescu, J.C. Mirza Rosca, Electrochemical and SEM studies of a new implant bioalloy in physiological electrolytes, Materials and Corrosion 60 (2009) 949-956.

[11] B.I. Wang, Y.F. Zheng, I.C. Zhao, Effects of Hf content and immersion time on electrochemical behavior of biomedical Ti-22Nb-xHf alloys in $0.9 \% \mathrm{NaCl}$ solution Materials and Corrosion 60 (2009) 330-335.

[12] B.I. Wang, Y.F. Zheng, I.C. Zhao, Electrochemical corrosion behavior of biomedical Ti-22Nb and Ti-22Nb-6Zr alloys in saline medium, Materials and Corrosion 60 (2009) 788-794.

[13] I. Milosev, T. Kosec, H. H. Strehblow, XPS and EIS study of the passive film formed on orthopaedic Ti-6Al-7Nb in Hank’s physiological solution, Electrochimica Acta 53 (2008) 35473558.

[14] S.A. Shabalovskaya, G.C. Rondelli, A.L. Undisz, J.W. Anderegg, T.D. Burleigh, M.E. Rettenmayr, The electrochemical characteristics of native Nitinol surfaces, Biomaterials 30 (2009) 3662-3671.

[15] Y. Okazaki, E. Gotoh, Comparison of metal release from various metallic biomaterials in vitro, Biomaterials 26 (2005) 11-21.

[16] N.J. Hallab, S. Anderson, M. Caicedo, A. Brasher, K. Mikecz, J.J. Jacobs, Effects of soluble metals on human peri-implant cells, Journal of Biomedical Materials Research 74A (2005) 124140. 
[17] S. Piazza, G. Lo Biundo, M. C. Romano, C. Sunseri, F. Di Quatro, In situ characterization of passive films on Al-Ti alloy by photocurrent and impedance, Corrosion Science 40 (1998) 1087-1108.

[18] S. Rao, Y. Okazaki, T. Tateishi, T. Ushida, Y. Ito, Cytocompatibility of new Ti alloy without Al and V by evaluating the relative growth ratios of fibroblasts L929 and osteoblasts MC3T3-E1 cells, Materials Science \& Engineering C 4 (1997) 311-314.

[19] W.F. Ho, C.P Ju, J.H. Chern Lin, Structure and properties of cast binary Ti-Mo alloys, Biomaterials 20 (1999) 2115-2122.

[20] G. He, J. Eckert, Q.L. Dai, M.L. Sui, W. Löser, M. Hagiwara, E. Ma, Nanostructured Ti-based multi-component alloys with potential for biomedical applications, Biomaterials 24 (2003) 5115-5120.

[21] M. Niinomi, Recent research and development in titanium alloys for biomedical applications and healthcare goods, Science \& Technology of Advanced Materials 4 (2003) 445-454.

[22] I. Halley-Demoulin, C. Valot, D. Ciosmak, M. Lallemant, J.J. Heizmann, C. Laruelle, Oxidation of titanium, zirconium and their alloys, texture of their oxide scales, Materials Science Forum 157-162 (1994) 521-528.

[23] C.V. D’Alkaine, L.M.M. Souza, F.C. Nart, The anodic behaviour of niobium - II. General experimental electrochemical aspects, Corrosion Science 34 (1993) 117-127.

[24] M.M. Lohrengel, Formation of ionic space charge layers in oxide films on valve metals, Electrochimica Acta 39 (1994) 1265-1271.

[25] N. Stojilovic, E.T. Bender, R.D. Ramsier, Surface chemistry of zirconium, Progress in Surface Science 78 (2005) 101-184.

[26] J. Fahey, D. Holmes, T.-L. Yau, Evaluation of localized corrosion of zirconium in acidic chloride solutions, Corrosion 53 (1997) 54-61.

[27] F. Rosalbino, D. Maccio, A. Saccone, E. Angelini, S. Delfino, Stability of the passive state of Zr-Nb crystalline alloys, Materials and Corrosion 63 (2012) 580-585.

[28] K.J. Bundy, in: Corrosion tests and standards: Application and interpretation, R. Baboian (Ed.); ASTM, Philadelphia, PA, 1995, p. 411.

[29] S.Y. Yu, J.R. Scully, Corrosion and passivity of Ti-13\% Nb-13\% Zr in comparison to other biomedical implant alloys, Corrosion 53 (1997) 965-976.

[30] D. Mareci, R. Chelariu, G. Ciurescu, D. Sutiman, T. Gloriant, Electrochemical aspects of Ti-Ta alloys in HBSS, Materials and Corrosion 61 (2010) 768-774. 
[31] D. Mareci, G. Ciurescu, R. Chelariu, I. Cretescu, D. Sutiman, In vitro corrosion study by electrochemical and surface analysis techniques of a Ti50Ta alloy for dental applications, Environ. Eng. Manag. J. 9 (2010) 81-87.

[32] B. Yeum, Electrochemical impedance spectroscopy: Data analysis software; Echem Software, Ann Arbor, 2001.

[33] B.E. Wilde, E. Williams, The use of current/voltage curves for the study of localized corrosion and passivity breakdown on stainless steels on chloride media, Electrochimica Acta 16 (1971) 1971-1985.

[34] J.R. Scully, R.G. Kelly, Methods for determining aqueous corrosion reaction rates, in: ASM Handbook, Vol. 13A, S.D. Cramer, B.S. Covino Jr. (Eds.); ASM International, Materials Park, OH, 2003, p. 68.

[35] G.S. Frankel, Pitting corrosion, in: ASM Handbook, Vol. 13A, S.D. Cramer, B.S. Covino Jr. (Eds.); ASM International, Materials Park, OH, 2003, p. 236.

[36] N.T.C. Oliveira, S.R. Biaggio, R.C. Rocha-Filho, N. Bocchi, Electrochemical studies on zirconium and its biocompatible alloys Ti-50Zr at.\% and $\mathrm{Zr}-2.5 \mathrm{Nb}$ wt.\% in simulated physiologic media, Journal of Biomedical Materials Research 74A (2005) 397-407.

[37] G. Rondelli, B.Vicentini, Effect of copper on the localized corrosion resistance of Ni-Ti shape memory alloy, Biomaterials 23 (2002) 639-644.

[38] J. Pan, D. Thierry, C. Leygraf, Electrochemical impedance spectroscopy study of the passive oxide film on titanium for implant application, Electrochimica Acta 41 (1996) 1143-1153.

[39] M. Aziz-Kerrzo, K.G. Conroy, A.M. Fenelon, S.T. Farrell, C.B. Breslin, Electrochemical studies on the stability and corrosion resistance of titanium-based implant materials, Biomaterials 22 (2001) 1531-1539.

[40] J. Pan, D. Thierry, C. Leygraf, Hydrogen peroxide toward enhanced oxide growth on titanium in PBS solution: Blue coloration and clinical relevance, Journal of Biomedical Materials Research 30 (1996) 393-402.

[41] J. Pan, D. Thierry, C. Leygraf, Electrochemical and XPS studies of titanium for biomaterial applications with respect to the effect of hydrogen peroxide, Journal of Biomedical Materials Research 28 (1994) 113-122.

[42] N.T.C. Oliveira, E.A. Ferreira, L.T. Duarte, S.R. Biaggio, R.C. Rocha-Filho, N. Bocchi, Corrosion resistance of anodic oxides on the Ti-50Zr and Ti-13Nb-13Zr alloys, Corrosion Science 51 (2006) 2068-2075. 
[43] F. Rosalbino, D. Maccio, P. Giannoni, R. Quarto, A. Saccone, Study of the in vitro corrosion behaviour and biocompatibility of Zr-2.5Nb and Zr-1.5Nb-1Ta (at\%) crystalline alloys, Journal of Materials Science: Materials in Medicine 22 (2011) 1293-1302.

[44] F. Mansfeld, Analysis and interpretation of EIS data for metals and alloys, Technical report 26; Solartron-Schlumberger, Hampshire, England, 1993, Chapter 4.

[45] M. Meticos-Hukovic, Z. Pilic, R. Babic, D. Omanovic, Influence of alloying elements on the corrosion stability of CoCrMo implant alloy in Hank’s solution, Acta Biomaterialia 2 (2006) 693-700.

[46] T.P. Moffat, R.M. Latanision, An electrochemical and X-ray photoelectron spectroscopy study of the passive state of chromium, J. Electrochem. Soc. 139 (1992) 1869-1879.

[47] S. Haupt, H.-H. Strehblow, Combined electrochemical and surface analytical investigations of the formation of passive layers, Corrosion Science 29 (1989) 163-182.

[48] E.B. Castro, J.R. Vilche, Investigation of passive layers on iron and iron-chromium alloys by electrochemical impedance spectroscopy, Electrochimica Acta 38 (1993) 1567-1572.

[49] G. Ciurescu, J. Izquierdo, J.J. Santana, D. Mareci, D. Sutiman, S. González, R.M. Souto, Characterization of the localized surface chemical activity of Ti-Mo and Ti-Ta alloys for biomedical applications using scanning electrochemical microscopy, International Journal of Electrochemical Science 7 (2012) 7404-7424.

Table 1. Chemical composition* and supplier of Ti and ZrTi samples.

\begin{tabular}{|l|l|c|}
\hline Sample & Composition / wt.\% & \multicolumn{1}{|c|}{ Supplier } \\
\hline Ti & Ti: 99.9 & IMNR $^{* *}$, Bucharest, Romania \\
Zr45Ti & Zr:55, Ti: 45 & Zirom S.A., Giurgiu, Romania \\
Zr25Ti & Zr: 75, Ti: 25 & Zirom S.A., Giurgiu, Romania \\
Zr5Ti & Zr: 95, Ti: 5 & Zirom S.A., Giurgiu, Romania \\
\hline
\end{tabular}

${ }^{*}$ The information included in the Table was supplied by the manufacturers.

** National Institute of Research \& Development for Non-ferrous and Rare Metals, Bucharest, Romania. 
Table 2. Electrochemical parameters determined from the cyclic polarization curves measured for Ti and ZrTi alloys in aerated Ringer's solution at $37^{\circ} \mathrm{C}$.

\begin{tabular}{|l|c|c|c|c|c|c|c|}
\hline Sample & $\begin{array}{c}E_{\text {corr }} / \\
\mathrm{V}_{\text {SHE }}\end{array}$ & $\begin{array}{c}j_{\text {corr }} / \\
\mathrm{nA} \mathrm{cm}\end{array}$ & $\begin{array}{c}j_{\text {pass }} / \\
\mu \mathrm{A} \mathrm{cm}\end{array}$ & $\begin{array}{c}E_{b p} / \\
\mathrm{V}_{\text {SHE }}\end{array}$ & $\begin{array}{c}E_{r p} / \\
\mathrm{V}_{\text {SHE }}\end{array}$ & $\begin{array}{c}E_{b p}-E_{\text {corr }} \\
/ \mathrm{mV}\end{array}$ & $\begin{array}{c}E_{r p}-E_{\text {corr }} \\
/ \mathrm{mV}\end{array}$ \\
\hline $\mathrm{Ti}$ & +0.056 & 560 & 36 & - & - & - & - \\
\hline Zr45Ti & -0.042 & 240 & 6.1 & +0.942 & +0.815 & 984 & 857 \\
\hline Zr25Ti & -0.075 & 125 & 0.9 & +0.527 & +0.455 & 602 & 530 \\
\hline Zr5Ti & -0.039 & 55 & 0.4 & +0.342 & +0.308 & 381 & 347 \\
\hline
\end{tabular}

Table 3. Impedance parameters of Ti and ZrTi alloys in aerated Ringer's solution at $37^{\circ} \mathrm{C}$.

\begin{tabular}{|c|c|c|c|c|c|c|}
\hline Sample & $\begin{array}{c}R_{c t} / \\
\mathrm{k} \Omega \mathrm{cm}^{2}\end{array}$ & $\begin{array}{c}10^{5} Q_{c t} / \\
\mathrm{S} \mathrm{cm}^{-2} s^{-n}\end{array}$ & $n_{c t}$ & $\begin{array}{c}R_{p L} / \\
\mathrm{k} \Omega \mathrm{cm}^{2}\end{array}$ & $\begin{array}{l}10^{5} Q_{p L} / \\
\mathrm{S} \mathrm{cm}^{-2} s^{-n}\end{array}$ & $n_{p L}$ \\
\hline & \multicolumn{6}{|c|}{$E=E_{c o r r}$} \\
\hline $\mathrm{Ti}$ & - & - & - & 500 & 1.2 & 0.81 \\
\hline Zr45Ti & - & - & - & 800 & 1.1 & 0.81 \\
\hline Zr25Ti & - & - & - & 1200 & 0.8 & 0.82 \\
\hline \multirow[t]{2}{*}{ Zr5Ti } & - & - & - & 2100 & 0.7 & 0.82 \\
\hline & \multicolumn{6}{|c|}{$E=+0.54 V \mathrm{SHE}$} \\
\hline $\mathrm{Ti}$ & 85 & 2.7 & 0.88 & 2900 & 1.0 & 0.85 \\
\hline Zr45Ti & 7 & 2.7 & 0.87 & 1400 & 0.9 & 0.82 \\
\hline Zr25Ti & 2 & 2.6 & 0.83 & 500 & 0.7 & 0.86 \\
\hline Zr5Ti & 31 & 2.4 & 0.85 & - & - & - \\
\hline
\end{tabular}


Table 4. Film thickness estimated from EIS results measured for Ti and ZrTi alloys in aerated Ringer's solution at $37^{\circ} \mathrm{C}$. A dielectric constant value of 65 was assumed.

\begin{tabular}{|l|c|}
\hline Sample & Film thickness / nm \\
\hline Ti & 4.5 \\
Zr45Ti & 3.3 \\
Zr25Ti & 3.2 \\
Zr5Ti & 2.9 \\
\hline
\end{tabular}

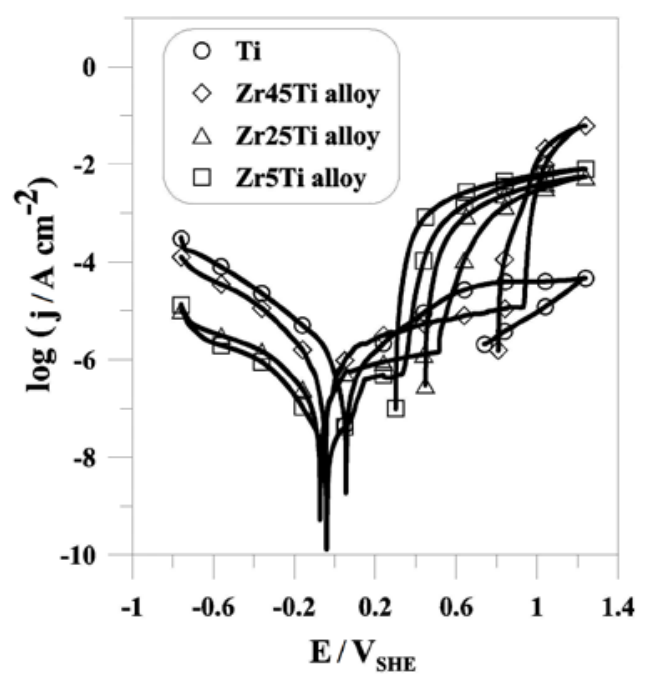

Figure 1. Cyclic potentiodynamic polarization curves of the $\mathrm{Ti}$ and $\mathrm{ZrTi}$ alloy samples in aerated Ringer's solution at $37^{0} \mathrm{C}$. Scan rate: $v=0.5 \mathrm{mV} / \mathrm{s}$.

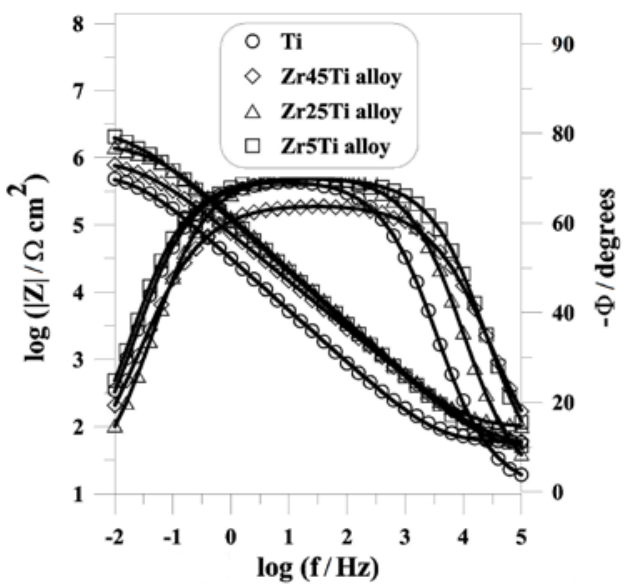

Figure 2. Measured (discrete points) and fitted (solid lines) impedance spectra typical for Ti and ZrTi alloy samples recorded at their open circuit potentials during exposure to aerated Ringer's solution at $37^{\circ} \mathrm{C}$. 


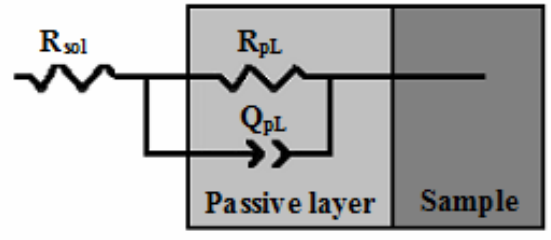

(A)

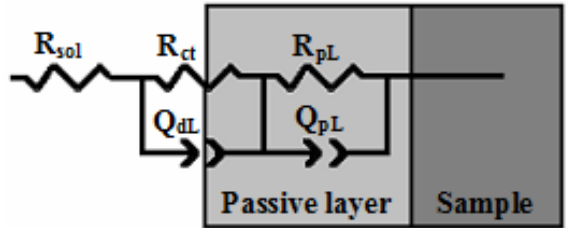

(B)|

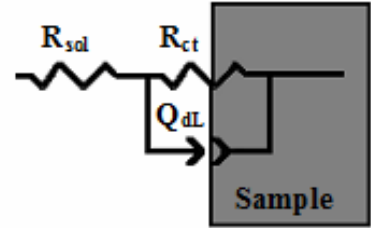

(C)

Figure 3.

Equivalent circuits (ECs) used for the interpretation of the measured impedance spectra: (A) onelayer model of a barrier-type compact oxide surface film with one time constant; (B) two-layer model of an sealed porous surface film with two time constants; and (C) simplified Randles' circuit of a corroding metal.

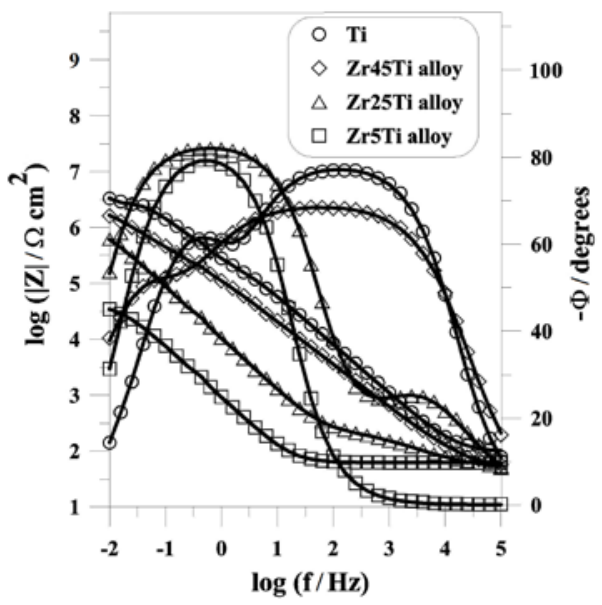

Figure 4.

Measured (discrete points) and fitted (solid lines) impedance spectra typical for $\mathrm{Ti}$ and $\mathrm{ZrTi}$ alloy samples polarized at +0.54 V SHE during exposure to aerated Ringer's solution at $37^{\circ} \mathrm{C}$.
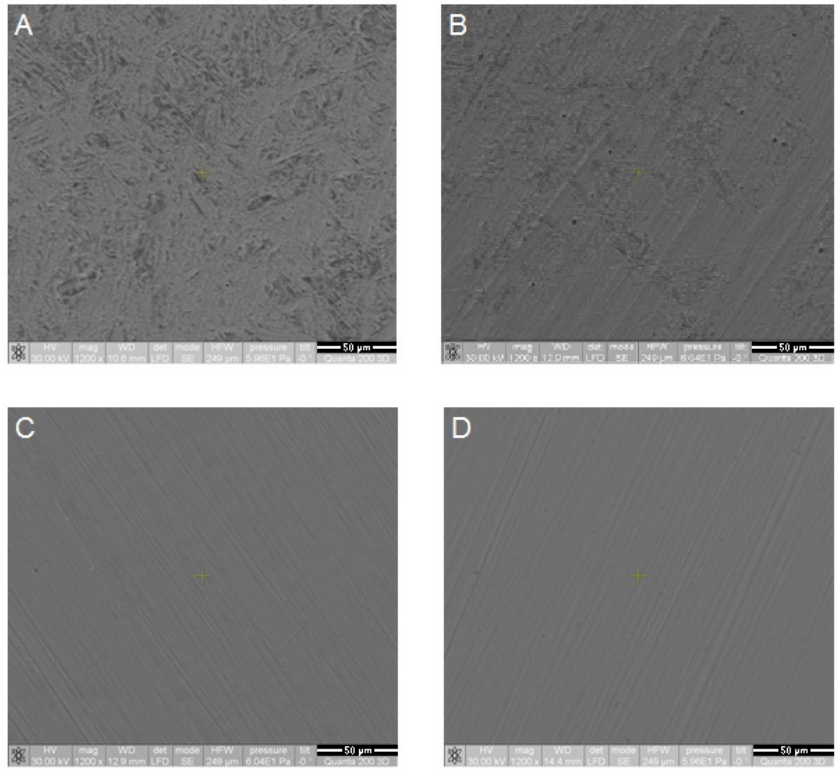

Figure 5.

Surface morphology of: (A) Zr5Ti alloy; (B) Zr25Ti alloy; (C) Zr45Ti alloy; and (D) Ti, after EIS tests at $+0.54 \mathrm{~V}_{\text {SHE }}$ in aerated Ringer's solution at $37{ }^{\circ} \mathrm{C}$. 

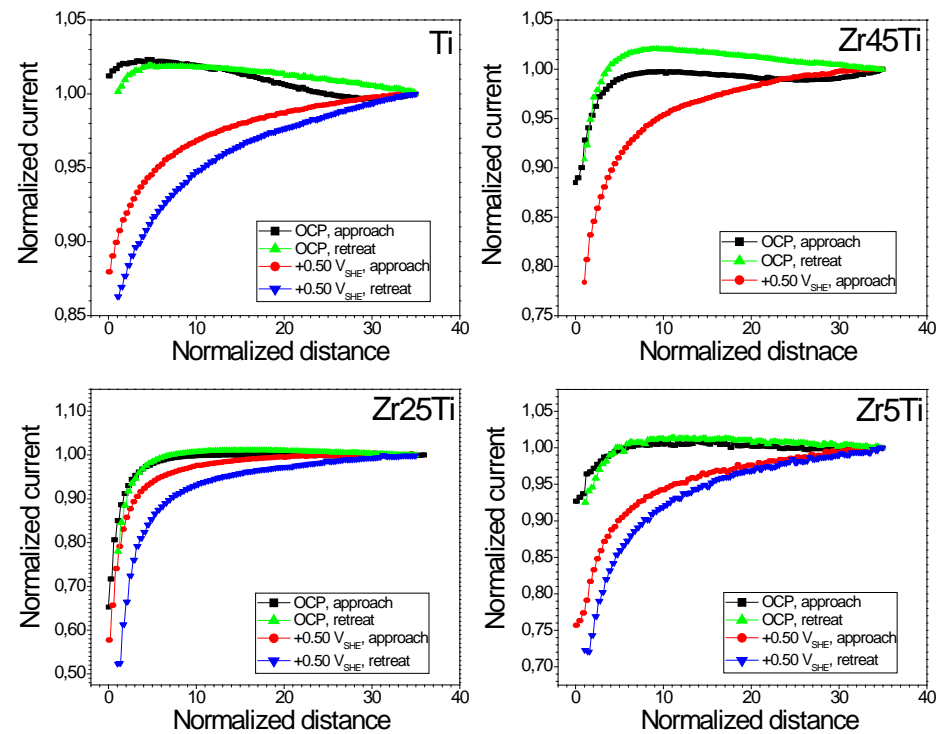

Figure 6.

Z-approach and Z-retreat curves measured with the Pt microelectrode above Ti and ZrTi alloys as the height of the tip is changed. The metallic samples are either left unbiased at their corresponding $E_{\text {corr }}$ or polarized at $+0.50 \mathrm{~V}_{\text {SHE }}$ during immersion in Ringer's $+0.5 \mathrm{mM}$ ferrocene-methanol solution. Tip potential: +0.70 Vshe. $i / i_{\text {lim }}$ is the dimensionless tip current, and $d / a$ is the dimensionless tipsubstrate distance.

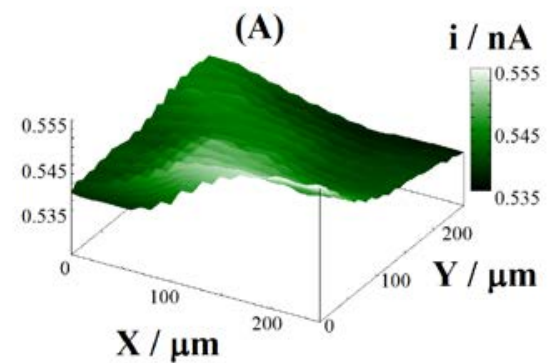

(C)

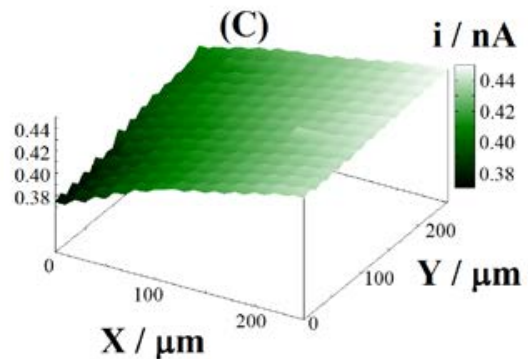

(B)

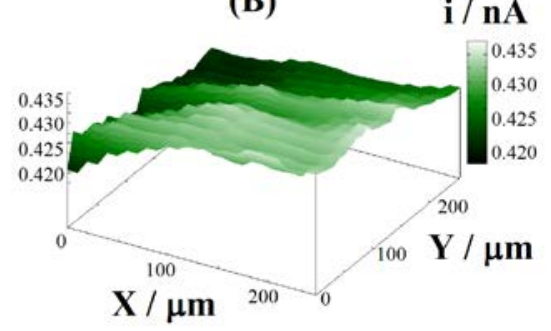

(D)

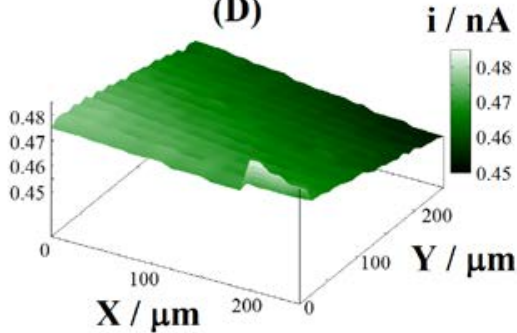

\section{Figure 7.}

SECM images of: (A) Ti, (B) Zr45Ti, (C) Zr25Ti, and (D) Zr5Ti samples, maintained at their respective $E_{\text {corr, }}$ during immersion in Ringer's $+0.5 \mathrm{mM}$ ferrocene-methanol solution. Tip potential: $+0.70 V_{\text {SHE }}$. Tip-substrate distance: $10 \mu \mathrm{m}$. Corresponding $i_{\text {lim }}$ values for the maps: (A) $0.53 \mathrm{nA}$, (B) $0.51 \mathrm{nA},(\mathrm{C}) 0.48 \mathrm{nA}$, and (D) $0.47 \mathrm{nA}$. 
(A)

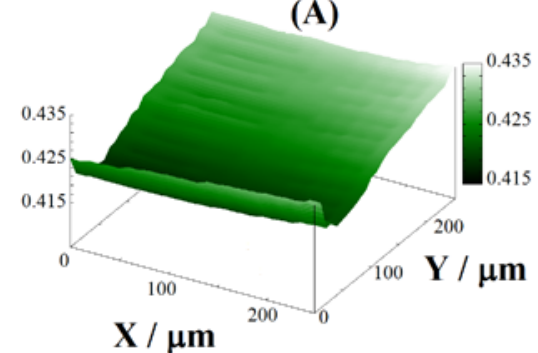

(C)

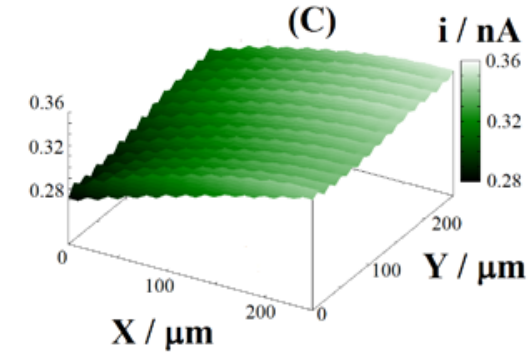

(B)

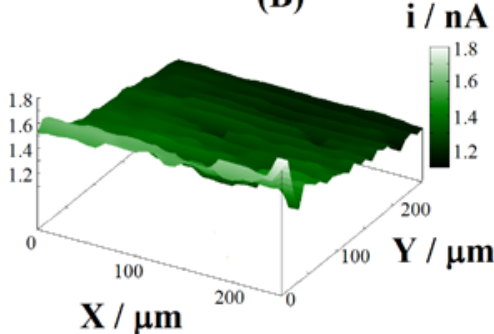

(D)

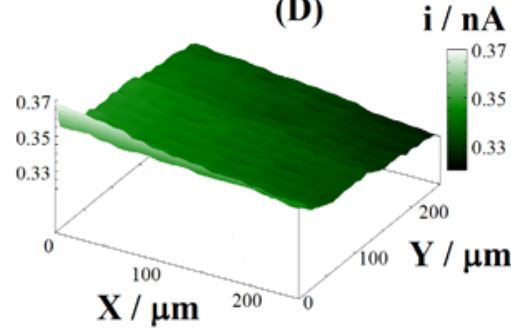

Figure 8.

SECM images of: (A) Ti, (B) Zr45Ti, (C) Zr25Ti, and (D) Zr5Ti samples, polarized at +0.50 Vshe, during immersion in Ringer's $+0.5 \mathrm{mM}$ ferrocene-methanol solution. Tip potential: +0.70 VsHE. Tip-

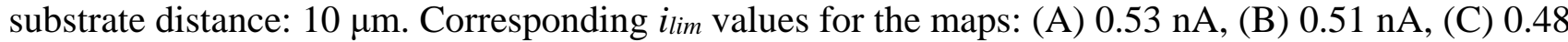
$\mathrm{nA}$, and (D) $0.47 \mathrm{nA}$.

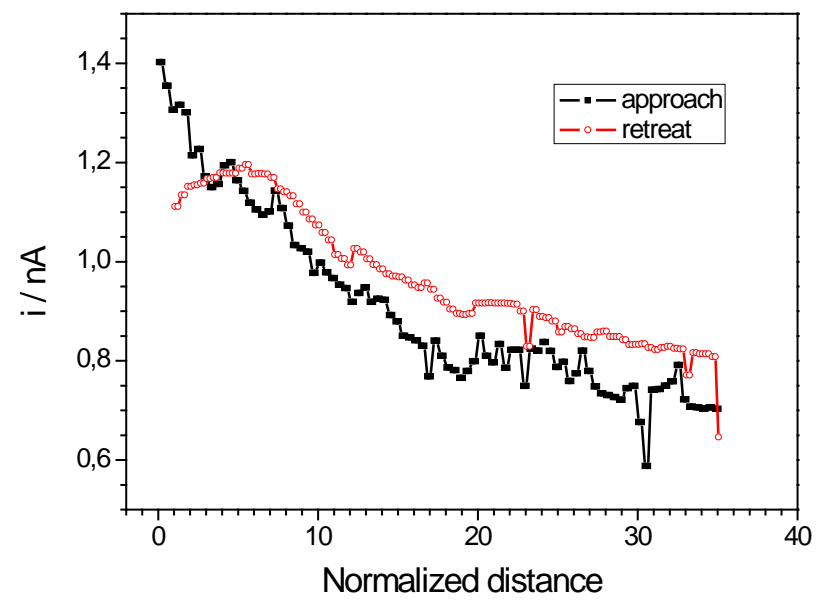

Figure 9.

Z-retreat and Z-approach curves measured with the Pt microelectrode above of Zr45Ti alloy after recording the SECM image shown in Figure 9B. The metallic sample was polarized at $+0.50 \mathrm{VsHE}$ during immersion in Ringer's $+0.5 \mathrm{mM}$ ferrocene-methanol solution. Tip potential: +0.70 VsHe. $i$ / $i_{\text {lim }}$ is the dimensionless tip current, and $d / a$ is the dimensionless tip-substrate distance. 